\title{
A Novel Dual-Band Left-Handed Metamaterial Design Method
}

\author{
Si Li, ${ }^{1}$ Wenhua Yu, ${ }^{2}$ Atef Z. Elsherbeni, ${ }^{3}$ Wenxing Li, ${ }^{1}$ and Yunlong Mao ${ }^{1}$ \\ ${ }^{1}$ College of Information and Communication Engineering, Harbin Engineering University, Harbin, Heilongjiang 150001, China \\ ${ }^{2} 2$ COMU, Inc., Fairfax, VA 22030, USA \\ ${ }^{3}$ Electrical Engineering Department, Colorado School of Mines, Golden, CO 80401, USA
}

Correspondence should be addressed to Wenxing Li; liwenxing@hrbeu.edu.cn

Received 3 February 2017; Revised 9 May 2017; Accepted 16 May 2017; Published 2 July 2017

Academic Editor: Shih Yuan Chen

Copyright (C) $2017 \mathrm{Si} \mathrm{Li} \mathrm{et} \mathrm{al.} \mathrm{This} \mathrm{is} \mathrm{an} \mathrm{open} \mathrm{access} \mathrm{article} \mathrm{distributed} \mathrm{under} \mathrm{the} \mathrm{Creative} \mathrm{Commons} \mathrm{Attribution} \mathrm{License,} \mathrm{which}$ permits unrestricted use, distribution, and reproduction in any medium, provided the original work is properly cited.

\begin{abstract}
A novel dual-band left-handed metamaterial (LHM) design method is proposed in this paper. Unlike other methods, where the designers focused their attentions on designing single LHM unit with multiple electric/magnetic resonances or combining multiple different LHM units together, the proposed method in this paper introduces an additional magnetic resonance to extract negative permeability, taking advantage of the areas between neighboring units. In this paper, we first designed a single-band single negative metamaterial $(\mu<0)$ and then connected neighboring units with metallic wires. This connection introduces a magnetic resonance that extracts another frequency band with negative permeability. With the help of arrayed metallic wires printed on the other side of the substrate, we successfully get a dual-band LHM. The proposed structures are analyzed with equivalent circuits and verified with simulations.
\end{abstract}

\section{Introduction}

Metamaterial (MTM) is a kind of engineering materials which exhibits exotic physical properties that are not encountered in nature. MTMs which have negative permittivity and permeability together are also referred to as left-hand metamaterials (LHMs). LHMs have a series of fantastic characteristics such as negative refractive index and backward waves, which draw great attention all over the world. MTMs have been successfully employed in many areas such as cloaking, absorbing materials $[1,2]$, and antenna designs $[3,4]$.

There are 2 kinds of realizations of metal-based LHMs in the microwave regime. The first kind of metal-based LHMs is implemented with magnetic resonances and arrayed metallic wires. The magnetic resonances, typically the split ring resonators (SRRs), are able to extract negative permeability at a specified frequency band, while the arrayed metallic wires are capable of providing negative permittivity at frequencies lower than their plasma frequency [5]. The other kind of LHMs is realized with the combination of electric LC (ELC) resonators $[6,7]$, which generate negative permittivity, and magnetic resonator. A common character of both LHMs is that the negative permeability is extracted from magnetic resonators.

Most of the proposed LHMs only have one left-handed (LH) passband. In contrast, there are only a few studies on multiband (MB) LHMs. Chen et al. [8] printed an extended "S shaped" metallic pattern on both sides of an FR4 substrate, which exhibits $2 \mathrm{LH}$ bands. However, for the purpose of containing multiple electric and magnetic resonances in a single unit, the cell size is relatively large. Wang et al. [9] stacked 2 layers of single-band (SB) LHM units with different geometrical dimensions to realize dual-band $\mathrm{LH}$ property but resulting in the reduction of transmission. Ekmekci et al. [10] stacked 3 layers of SRRs with different number of splits to extract two single negative $(\mu<0)$ bands but the transmission character in this work is also not good. Turkmen et al. [11] proposed a nested "U-shape" resonator that exhibits two or three LH bands according to the number of "U-shapes." However, neither the electric nor the magnetic resonances are strong enough that the LH bandwidth is narrow and the $\mathrm{LH}$ transmission character is bad. Xu et al. [12] proposed a fractal "tree-shape" pattern featuring 3 magnetic resonances and an electric resonance apart from the lower metal plasma response. This complex structure is verified to have $2 \mathrm{LH}$ 


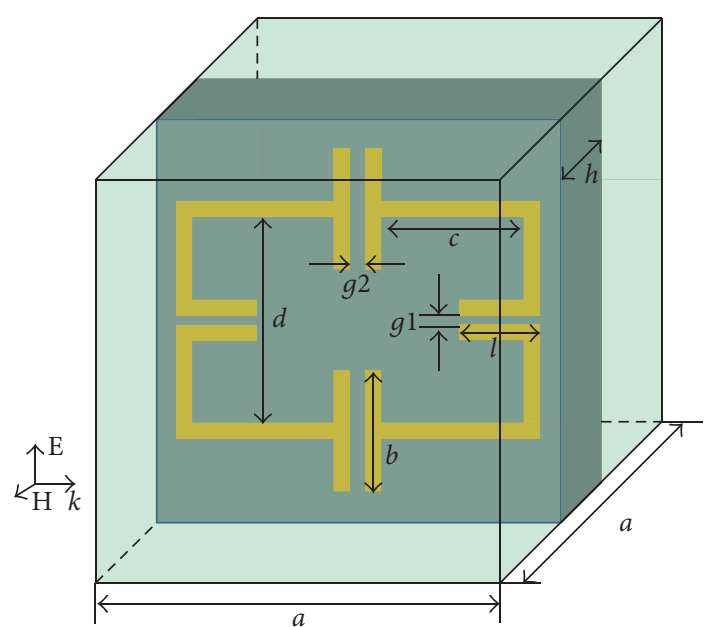

FIGURE 1: Geometry illustration of the proposed dual-band SNG.

bands with measurements. Liu et al. [13] demonstrated a complex nonlinear dual-band LHM which is geometrically similar to that proposed in [8]. Zhou et al. [14] proposed a double "Z-shaped" structure which exhibits $2 \mathrm{LH}$ passbands under parallel incidence and $3 \mathrm{LH}$ passbands under normal incidence.

It can be concluded from the above-mentioned works that the main challenge facing MB LHMs is how to excite multiple magnetic resonances. In this work, we propose a novel method to design a DB LHM. First of all, we designed a single-band (SB) MTM with only $\mu<0$. Then we tactfully took advantage of the area between neighboring MTM units to introduce an additional magnetic resonance. Thereby, we get a DB MTM, which can be easily turned into a DB LHM by printing metallic wires on the other side of the substrate [15]. The proposed structures are analyzed with equivalent circuits and verified with simulations. This method could be important guidance in the design of MB LHMs.

\section{Single Negative Metamaterial}

First of all, we designed a single-band single negative metamaterial (SNG) only with negative permeability. The geometry of the proposed structure is displayed in Figure 1, where we have $a=5 \mathrm{~mm}, b=1.5 \mathrm{~mm}, c=1.85 \mathrm{~mm}, d=3 \mathrm{~mm}, g 1$ $=0.1 \mathrm{~mm}, g 2=0.1 \mathrm{~mm}$, and $l=1 \mathrm{~mm}$. The width of the lines is $w=0.2 \mathrm{~mm}$, and the thickness is $t=0.018 \mathrm{~mm}$. This pattern is printed on a $0.4 \mathrm{~mm}$ thick square FR4 substrate which has $\varepsilon_{r}=4.2$.

2.1. Equivalent Circuit Analysis. The equivalent circuit for this structure is displayed in Figure 2.

According to strip line theory [16], the inductance of a strip line can be calculated using

$$
L=2 \times 10^{-4} l\left[\ln \left(\frac{l}{w+t}\right)+1.193+0.2235 \frac{w+t}{l}\right],
$$

where $l$ is the length of the strip line, $w$ is the width, and $t$ is the thickness. Assume that we have a function,

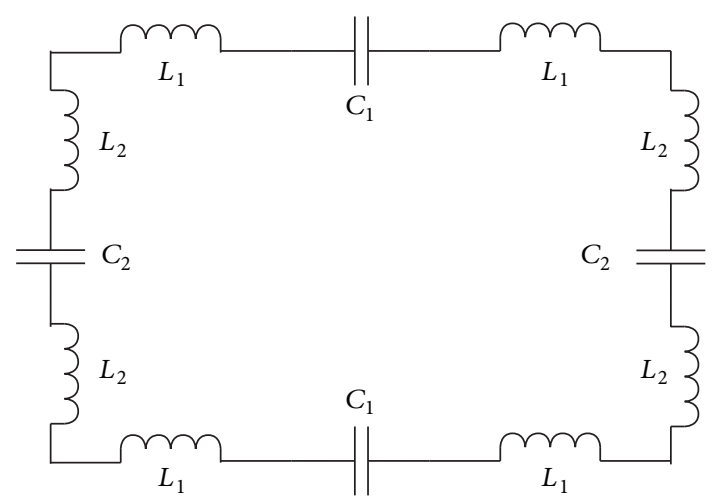

FIGURE 2: Equivalent circuit of the structure in Figure 1.

"Cal_induc $(w, l, t)$," to represent $(1)$; then the inductance can be calculated as

$$
L_{1}+L_{2}=\text { Cal_induc }\left(w, c+\frac{d}{2}-w-\frac{g 1}{2}+l, t\right),
$$

which is $3.4 \mathrm{nH}$. The total inductance for this circuit is $L_{m 1}=$ $4\left(L_{1}+L_{2}\right)=13.6 \mathrm{nH}$.

The capacitance per unit length of paralleled strip lines is calculated using $[16,17]$

$$
C_{\mathrm{pul}}=\varepsilon_{0} \varepsilon_{e} F(k),
$$

where $\varepsilon_{0}$ is the permittivity of free space; $\varepsilon_{e}$ and $F(k)$ are calculated as

$$
\begin{aligned}
& \varepsilon_{e}=1+\frac{\left(\varepsilon_{r}-1\right) F(k)}{2 F(k 1)}, \\
& F(k)= \begin{cases}\frac{1}{\pi} \ln \left(2 \frac{1+\sqrt{k^{\prime}}}{1-\sqrt{k^{\prime}}}\right), & 0<k \leq \frac{1}{\sqrt{2}}, \\
\pi \ln \left(2 \frac{1+\sqrt{k}}{1-\sqrt{k}}\right)^{-1}, & \frac{1}{\sqrt{2}}<k \leq 1,\end{cases}
\end{aligned}
$$

where $k=a / b, a=g 2 / 2, b=a+w 3, k^{\prime}=\sqrt{1-k^{2}}$, and $k 1=$ $\sinh (\pi a / 2 h) / \sinh (\pi b / 2 h)$. Assume that there is a function, "Cal_cap $\left(w, g, h, \varepsilon_{r}\right)$," to represent (3), where $w$ is the width and $g$ is the gap of the paralleled wires, $h$ is the thickness of the substrate, and $\varepsilon_{r}$ is the relative permeability of the substrate; then we will have

$$
\begin{aligned}
& C_{1}=b \times \text { Cal_cap }\left(w, g 2, h, \varepsilon_{r}\right), \\
& C_{2}=l \times \text { Cal_cap }\left(w, g 1, h, \varepsilon_{r}\right) .
\end{aligned}
$$

It can be calculated that $C_{1}=0.06 \mathrm{pf}$ and $C_{2}=0.04 \mathrm{pf}$. Hence, the total capacitance is

$$
C_{m 1}=\frac{C_{1} C_{2}}{2\left(C_{1}+C_{2}\right)} .
$$

The resonant frequency is calculated using

$$
f_{m 1}=\frac{1}{2 \pi \sqrt{L_{m 1} C_{m 2}}}
$$

which is $12.1 \mathrm{GHz}$. 
2.2. Verification with Simulations. To verify the single negative property of this structure, we ran a full wave simulation using HFSS version 15. The simulation domain and the incidence of a plane wave are illustrated in Figure 1. The polarization of the electric field and magnetic field is in the vertical and horizontal directions, respectively. The light green box is an air box. The top and bottom boundaries are defined as PEC, while the front and back boundaries are defined as PMC. The simulated $S$ parameters are displayed in Figure 3.

As is known to all, SNGs are not capable of power transmission. In Figure 3, $S_{21}$ reaches a peak at $12.8 \mathrm{GHz}$, indicating that single negative property may happen there. The effective parameters retrieved using " $S$ parameter retrieval" method $[18,19]$ are displayed in Figure 4. Negative permeability can be observed at a frequency range from $12.18 \mathrm{GHz}$ to $13.37 \mathrm{GHz}$, while the permittivity stays positive in the discussed frequency spectrum. The magnetic resonant frequency is $12.8 \mathrm{GHz}$, which is a little higher than our numerical analysis. The absolute single negative bandwidth is $1.19 \mathrm{GHz}$ and its relative bandwidth is $9.3 \%$.

\section{Dual-Band LHM Implementation}

In the numerical analysis of split ring resonators (SRRs) [20], the effective permeability is closely related to a coefficient, " $F$," which is the fractional area of a unit cell occupied by the interior of the split ring. The larger " $F$ " is, the stronger the magnetic resonance will be. Therefore, in most designs, " $F$ " is set up very close to 1 , which overrode the fact that the area between neighboring units can also be taken advantage of to build additional magnetic resonance. Particularly, when this area is large enough, the corresponding magnetic resonance should also be strong enough to extract negative permeability. Hence, following the design principle in [21] which claimed that the equivalent circuit for a magnetic resonator should be mirror-symmetry single-loop, we introduced an additional magnetic resonance easily by connecting the neighboring SNG units with metallic wires. The updated structure is displayed in Figure 5, where we have two neighboring units in the "E" direction connected. The pattern beyond the blue dashed line, which is referred to as "R1," is the original magnetic resonant unit, while the pattern beyond the red dashed line, which is referred to as " $\mathrm{R} 2$," is the newly built magnetic resonant unit.

3.1. Equivalent Circuit Analysis. In the previous discussion, we already analyzed the equivalent circuit of the original magnetic resonator, "R1." Without the consideration of interactions between "R1" and "R2," we can also analyze "R2" with equivalent circuit. The equivalent circuit is displayed in Figure 6.

Following (1), the inductance can be calculated as

$$
2 L_{1}+L_{3}=\text { Cal_induc }(w, 2 c+a-d, t),
$$

which is $5.1 \mathrm{nH}$. The total inductance for this circuit is $L_{m 2}=$ $2\left(2 L_{1}+L_{3}\right)=10.2 \mathrm{nH}$, while the total capacitance is $C_{m 2}=$ $C_{1} / 2=0.032 \mathrm{pF}$. Hence, the resonant frequency is $8.84 \mathrm{GHz}$.

This connection also brings an electric resonance. Since the domain size is much smaller than the wavelength of the incidence, the external electric field across a unit can be approximated as $U=E_{z} a=R I+(-j \omega L) I+I /(-j \omega C)$, where $E_{z}$ is the external electric field per length, $I$ is the induced current, $R$ and $L$ are the total parasitic resistance and inductance, respectively, and $C$ is the total capacitance by the gap. Then, the volume current density $J_{v}$ in each unit can be homogenized as

$$
J_{v}=\frac{I}{a^{2}}=\frac{j \omega /(a L)}{\omega^{2}-1 /(L C)+j \omega R / L} E_{z}=\sigma^{\prime} E_{z} .
$$

Meanwhile [22]

$$
\varepsilon_{\mathrm{eff}}=1+j \frac{\sigma^{\prime}}{\omega \varepsilon_{0}}
$$

Hence,

$$
\varepsilon_{\mathrm{eff}}=1-\frac{\left[\omega^{2}-1 /(L C)\right] /\left(a L \varepsilon_{0}\right)-j \omega R /\left(a L^{2} \varepsilon_{0}\right)}{\left[\omega^{2}-1 /(L C)\right]^{2}+(\omega R / L)^{2}} .
$$

From (11), at the resonant frequency, the real part of the effective permittivity is 1 . With the increase of the frequency, the real part of the effective permittivity is getting smaller and it may be negative at frequencies higher than the resonant frequency. The equivalent circuit for this electric resonator is illustrated in Figure 7.

The inductance can be approximated as

$$
L_{4}=\text { Cal_induc }\left(w, \frac{a}{2}-\frac{g 1}{2}-w+l, t\right),
$$

which is $2.54 \mathrm{nH}$. Hence, the electric resonant frequency is $10.8 \mathrm{GHz}$.

By now, both the magnetic resonances and the electric resonator have been independently analyzed with equivalent circuits. From the numerical analysis, we can predict that this structure exhibits single negative property at frequencies around $8 \mathrm{GHz}$ and double negative property at frequencies around $12 \mathrm{GHz}$. Further verifications are still needed through simulations.

3.2. Verification with Simulations. The simulation is also carried out with HFSS. The set-up for the simulation is exactly the same as before. The simulated $S$ parameters are displayed in Figure 8, and the corresponding retrieved effective parameters are displayed in Figures 9(a)-9(d).

In Figure 8, there are 2 passbands where $S_{11}$ is smaller than $-10 \mathrm{~dB}$, and the peaks happen at around $7 \mathrm{GHz}$ and $12 \mathrm{GHz}$, respectively. A strong decrease of $S_{21}$ can be observed at frequencies around $8 \mathrm{GHz}$, which identifies that power can hardly transmit at these frequencies.

It can be observed from Figure 9 that the real part of permittivity is negative at frequencies higher than $11.33 \mathrm{GHz}$ in the discussed frequency range. On the other hand, the real part of permeability is negative at two frequency spectrums, from $7.33 \mathrm{GHz}$ to $8.57 \mathrm{GHz}$ and from $11.58 \mathrm{GHz}$ to $13.68 \mathrm{GHz}$. Therefore, this structure exhibits single negative property at frequencies from $7.33 \mathrm{GHz}$ to $8.57 \mathrm{GHz}$ and a $\mathrm{LH}$ property at frequencies from $11.58 \mathrm{GHz}$ to $13.68 \mathrm{GHz}$.

This DB MTM can be easily turned into a DB LHM if we print arrayed metallic wires with higher plasma frequency than $8.57 \mathrm{GHz}$ on the other side of the substrate [14]. 


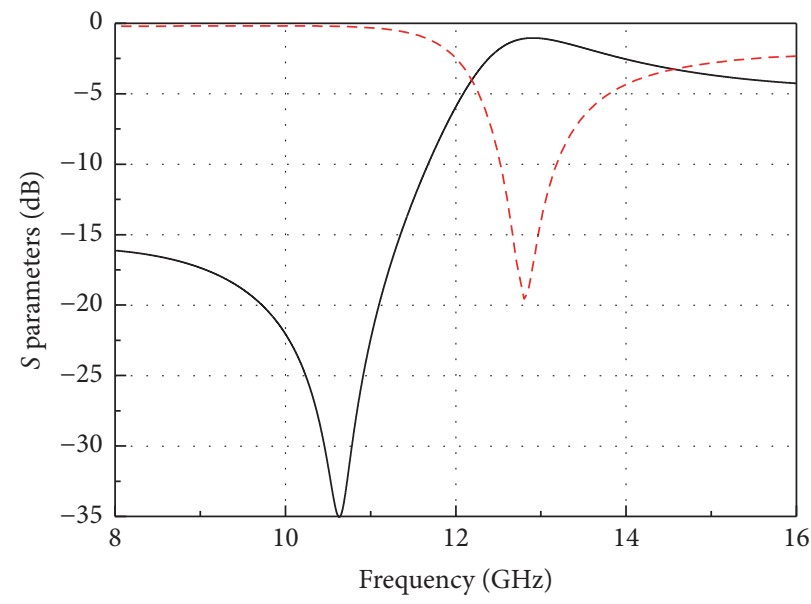

$-S_{11}$
$--S_{21}$

FIGURE 3: The simulated $S$ parameters.

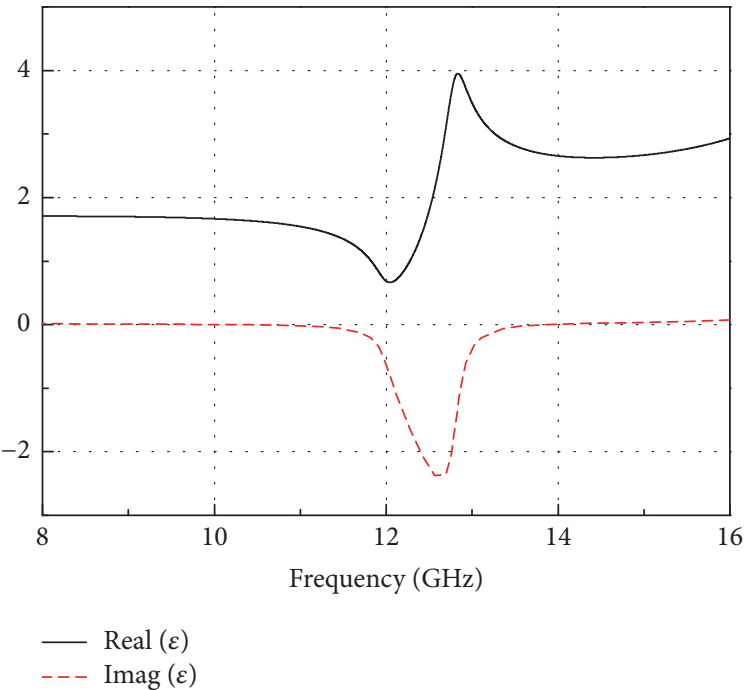

(a)

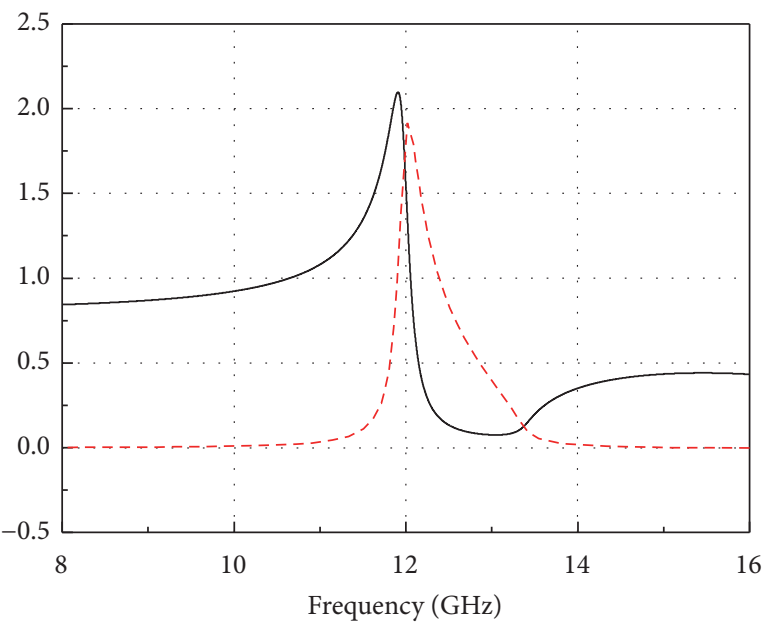

- Real $(Z)$

- - - $\operatorname{Imag}(Z)$

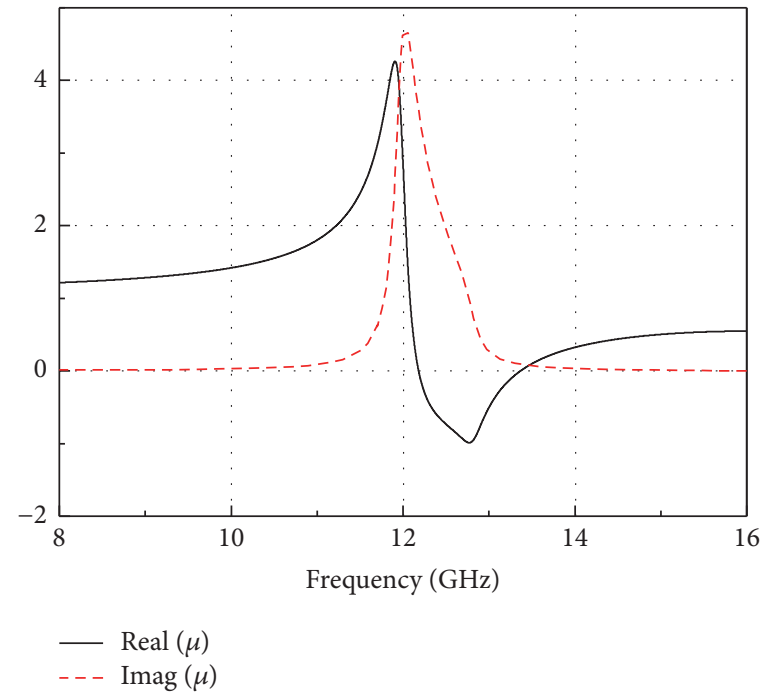

(b)

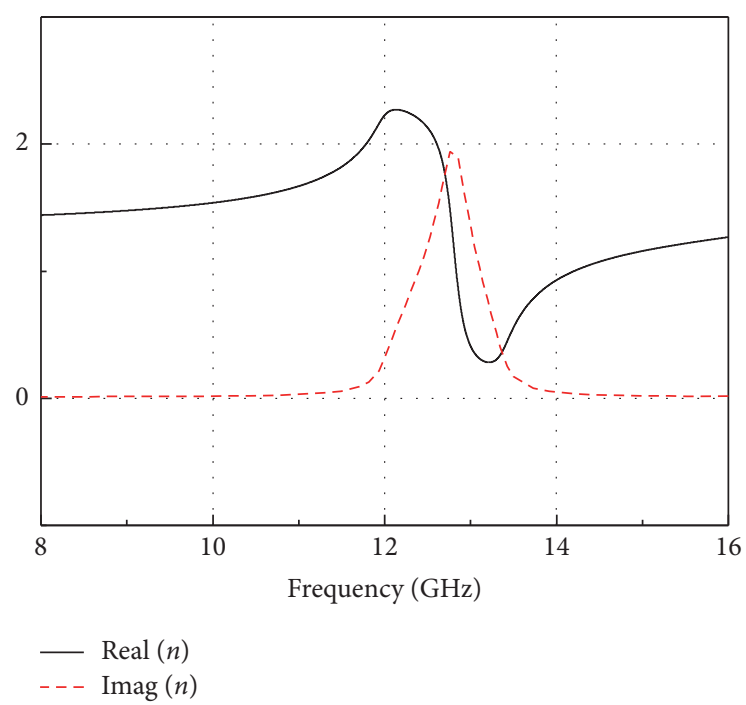

(d)

FIGURE 4: Retrieved effective (a) permittivity “ $\varepsilon$," (b) permeability “ $\mu$," (c) impedance " $Z$," and (d) refractive index " $n$.” The black solid lines refer to the real part of these parameters, while the red dashed lines refer to the imaginary part. 


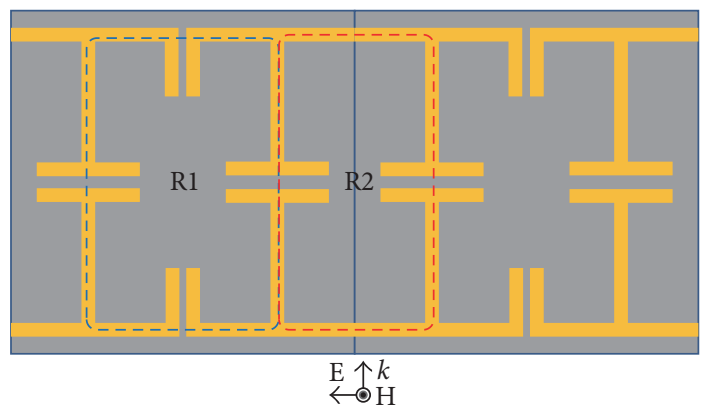

FIGURE 5: Metallic structure of the dual-band MTM.

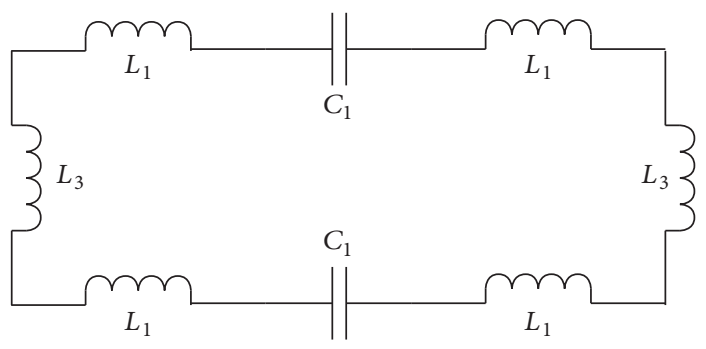

FIgURE 6: Equivalent circuit for "R2."

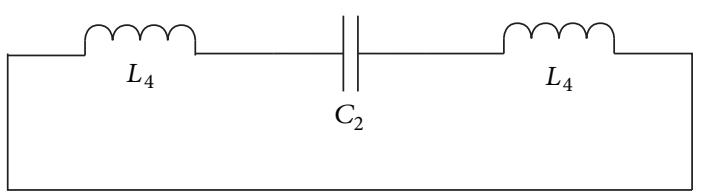

FIGURE 7: Equivalent circuit of electric resonator.

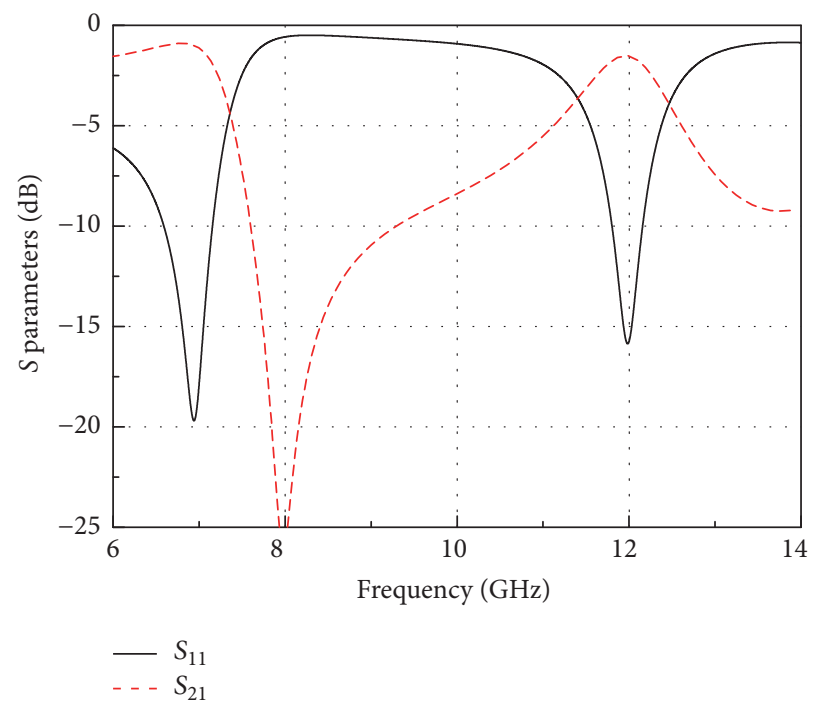

FIGURE 8: Simulated $S$ parameters of the proposed structure.

3.3. Dual-Band LHM Implementation. An optimized structure of the metallic wires printed on the other side of the substrate is displayed in Figure 10, where $e=3.4 \mathrm{~mm}$ and $f$ $=0.4 \mathrm{~mm}$.
The simulated $S$ parameters for the updated structure are displayed in Figure 11, and the corresponding effective parameters are displayed in Figure 12. Double negative properties are observed at frequency ranges approximately 


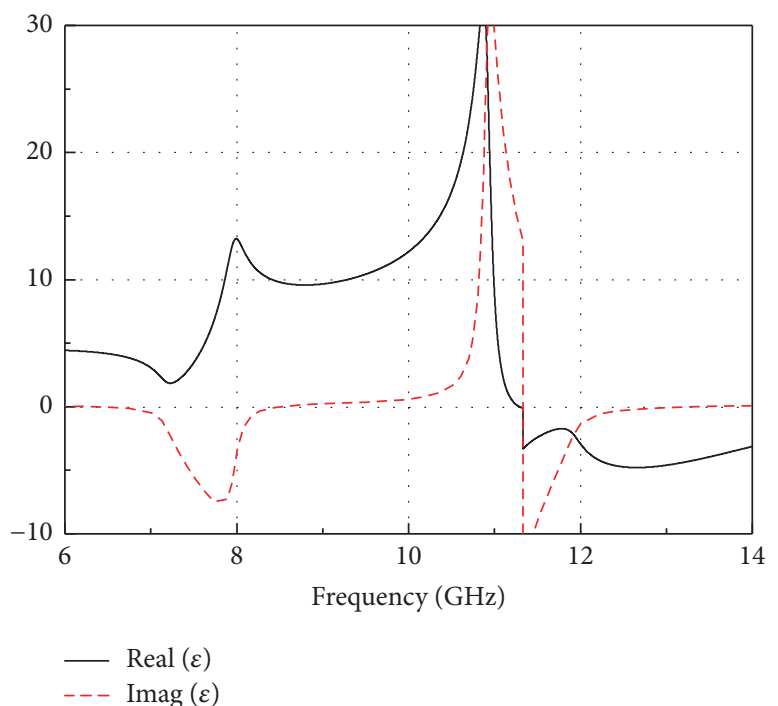

(a)

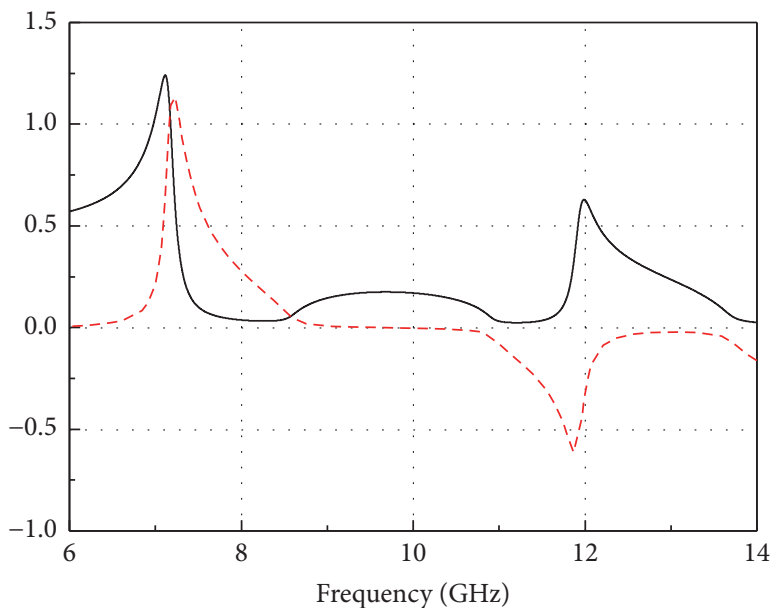

- Real $(Z)$

- . - $\operatorname{Imag}(Z)$

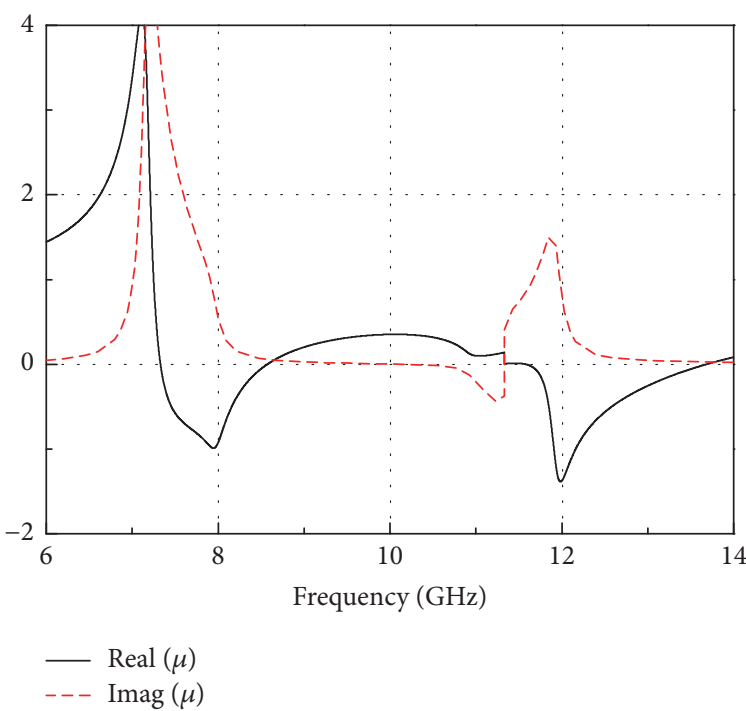

(b)

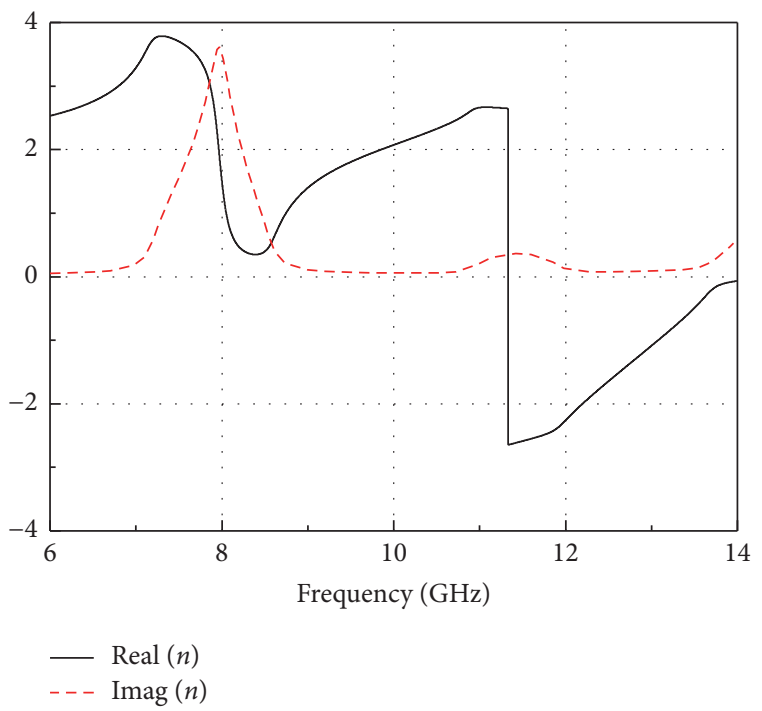

(d)

Figure 9: Retrieved effective (a) permittivity “ $\varepsilon$," (b) permeability “ $\mu$," (c) impedance " $Z$," and (d) refractive index " $n$.” The black solid lines refer to the real part of these parameters, while the red dashed lines refer to the imaginary part.

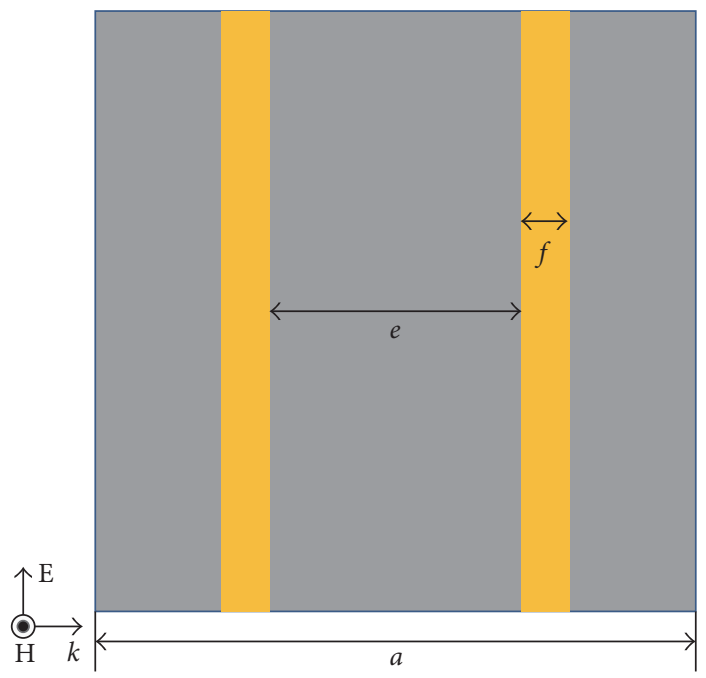

FIGURE 10: Geometry of the metallic wires printed on the other side of the substrate. 


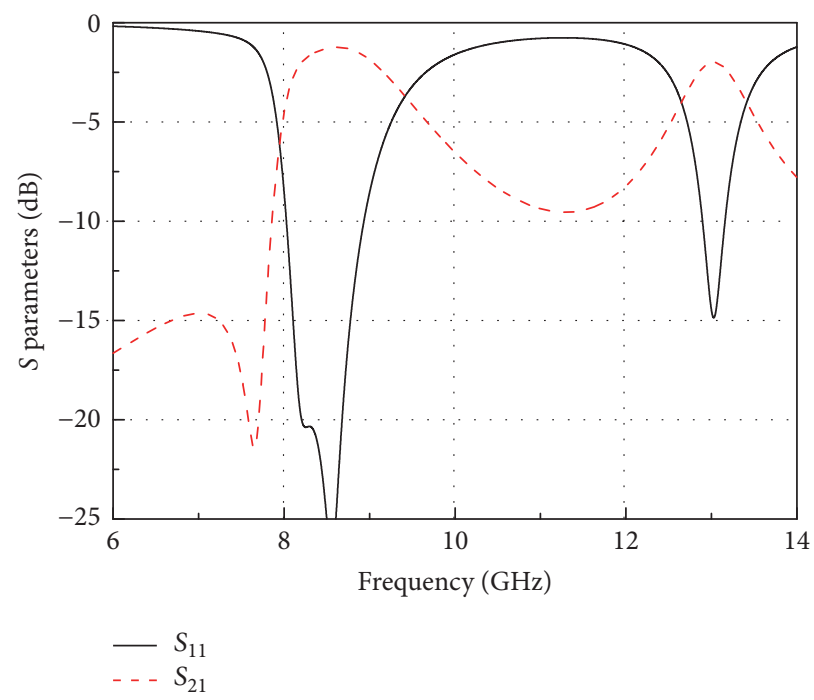

FIGURE 11: Simulated $S$ parameters of the DB LHM.

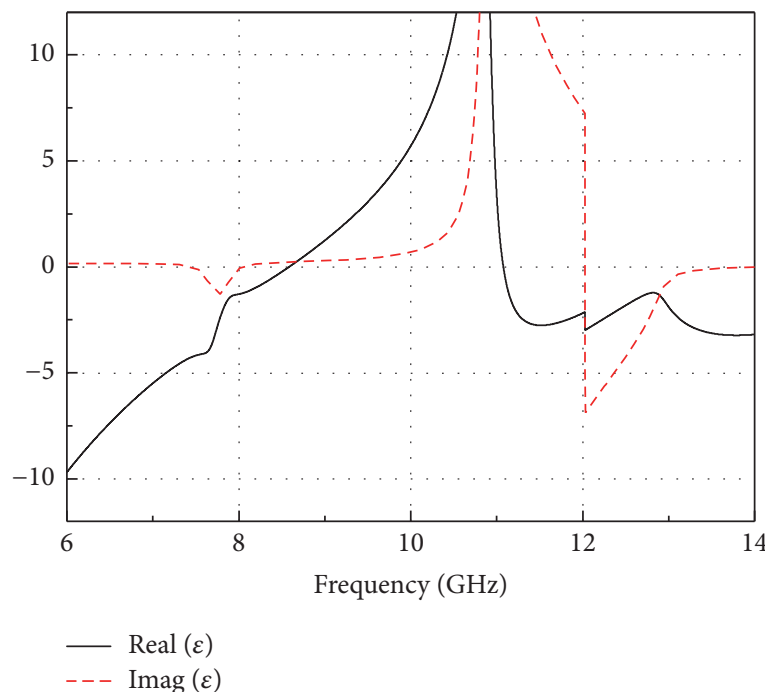

(a)

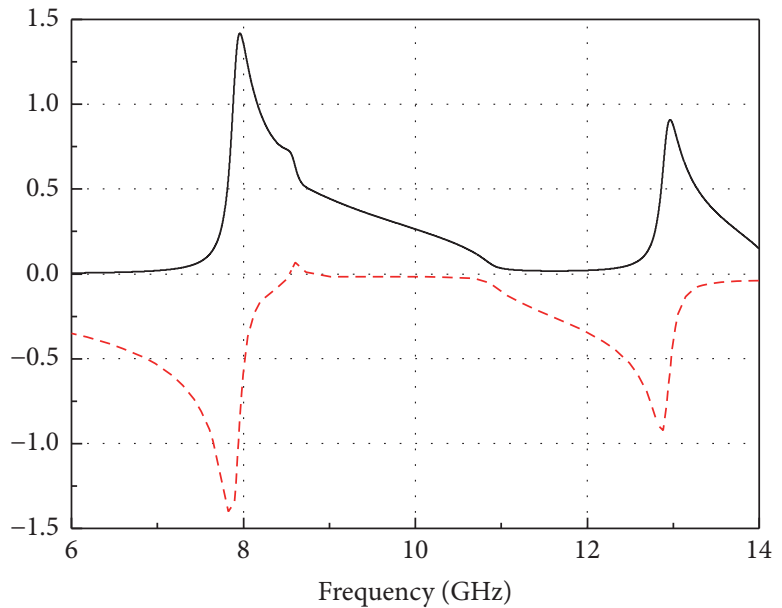

$-\operatorname{Real}(Z)$

- - $\operatorname{Imag}(Z)$

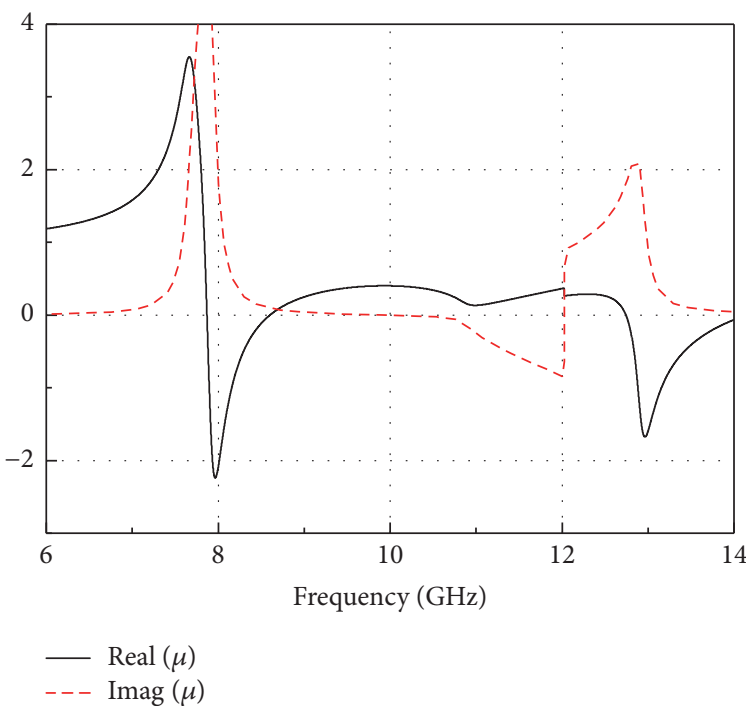

(b)

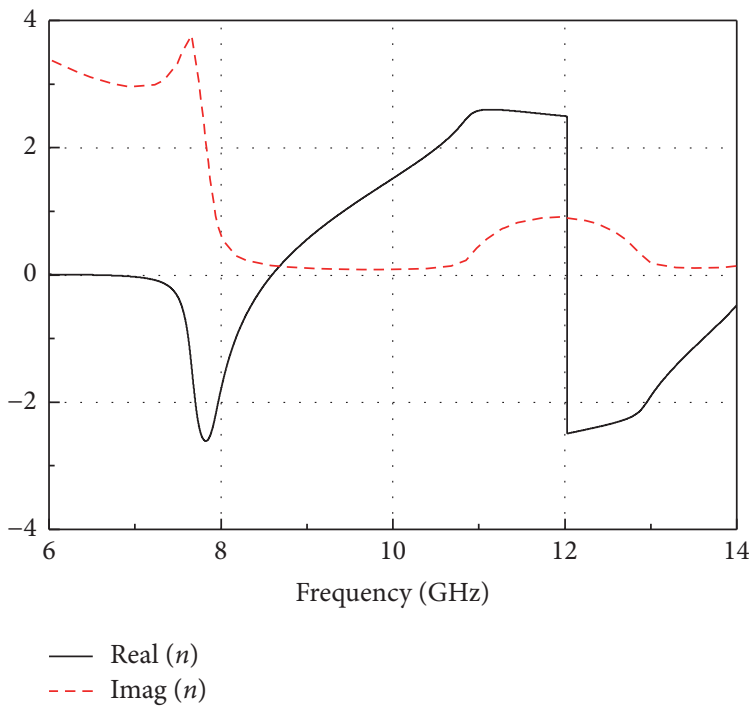

(d)

FIGURE 12: Retrieved effective (a) permittivity “ $\varepsilon$," (b) permeability “ $\mu$,” (c) impedance " $Z$," and (d) refractive index " $n$." The black solid lines refer to the real part of these parameters, while the red dashed lines refer to the imaginary part. 
from $7.87 \mathrm{GHz}$ to $8.60 \mathrm{GHz}$ and from $12.74 \mathrm{GHz}$ to $14 \mathrm{GHz}$. The corresponding relative bandwidths are $8.8 \%$ and $9.4 \%$, respectively.

The mutual coupling between the metallic structures on both sides of the substrate decreases the equivalent inductance, causing both the magnetic resonances and the electric resonance to shift to higher frequencies and also the reduction of the MTM bandwidth.

\section{Conclusion}

In this paper, we introduced a novel method for the design of dual-band LHM. This design method is explained step by step from a single-band single negative metamaterial $(\mu<0)$ to a dual-band LHM. The key point for this method is the introduction of an additional magnetic resonance, which is skillfully operated taking advantage of the areas between neighboring units. Metallic structures that appeared in our design procedure are well analyzed with equivalent circuits and verified with simulations. This method has an important meaning in the design of multiband LHMs.

\section{Conflicts of Interest}

The authors declare that there are no conflicts of interest regarding the publication of this paper.

\section{Acknowledgments}

This paper is supported by the Foundational Research Funds for the Central Universities (HEUCFD1433).

\section{References}

[1] Y. J. Yoo, H. Y. Zheng, Y. J. Kim et al., "Flexible and elastic metamaterial absorber for low frequency, based on small-size unit cell," Applied Physics Letters, vol. 105, no. 4, Article ID 041902, 2014.

[2] J. Hao, É. Lheurette, L. Burgnies, É. Okada, and D. Lippens, "Bandwidth enhancement in disordered metamaterial absorbers," Applied Physics Letters, vol. 105, no. 8, Article ID 081102, 2014.

[3] R. K. Saraswat and M. Kumar, "Miniaturized slotted ground UWB antenna loaded with metamaterial for WLAN and WiMax applications," Progress In Electromagnetics Research B, vol. 65, pp. 65-80, 2016.

[4] S. Yan, P. J. Soh, and G. A. E. Vandenbosch, "Compact All-Textile Dual-Band Antenna Loaded with MetamaterialInspired Structure," IEEE Antennas and Wireless Propagation Letters, vol. 14, pp. 1486-1489, 2015.

[5] J. B. Pendry, A. J. Holden, W. J. Stewart, and I. I. Youngs, "Extremely low frequency plasmons in metallic mesostructures," Physical Review Letters, vol. 76, pp. 4773-4776, 1996.

[6] R. Liu, A. Degiron, J. J. Mock, and D. R. Smith, "Negative index material composed of electric and magnetic resonators," Applied Physics Letters, vol. 90, no. 26, Article ID 263504, 2007.

[7] D. Schurig, J. J. Mock, and D. R. Smith, "Electric-field-coupled resonators for negative permittivity metamaterials," Applied Physics Letters, vol. 88, no. 4, Article ID 041109, 2006.
[8] H. Chen, L. Ran, J. Huangfu et al., "Metamaterial exhibiting lefthanded properties over multiple frequency bands," Journal of Applied Physics, vol. 96, no. 9, pp. 5338-5340, 2004.

[9] J. Wang, S. Qu, Y. Yang, H. Ma, X. Wu, and Z. Xu, "Multiband left-handed metamaterials," Applied Physics Letters, vol. 95, no. 1, p. 014105, 2009.

[10] E. Ekmekci, K. Topalli, T. Akin, and G. Turhan-Sayan, "A tunable multi-band metamaterial design using micro-split SRR structures," Optics Express, vol. 17, no. 18, pp. 16046-16058, 2009.

[11] O. Turkmen, E. Ekmekci, and G. Turhan-Sayan, "Nested Uring resonators: A novel multi-band metamaterial design in microwave region," IET Microwaves, Antennas and Propagation, vol. 6, no. 10, pp. 1102-1108, 2012.

[12] H.-X. Xu, G.-M. Wang, C.-X. Zhang et al., "Multi-band lefthanded metamaterial inspired by tree-shaped fractal geometry," Photonics and Nanostructures - Fundamentals and Applications, vol. 11, no. 1, pp. 15-28, 2013.

[13] Y. Liu, X. Zhou, K. Song et al., "Quasi-phase-matching of the dual-band nonlinear left-handed metamaterial," Applied Physics Letters, vol. 105, no. 20, 2014.

[14] H. Zhou, C. Wang, and H. Peng, "A novel double-incidence and multi-band left-handed metamaterials composed of double Z-shaped structure," Journal of Materials Science: Materials in Electronics, vol. 27, no. 3, pp. 2534-2544, 2016.

[15] R. A. Shelby, D. R. Smith, and S. Schultz, "Experimental verification of a negative index of refraction," Science, vol. 292, no. 5514, pp. 77-79, 2001.

[16] I. Bahl and P. Bhartia, Microwave Solid State Circuit Design, Wiley, 2003.

[17] S. Gevorgian and H. Berg, "Line capacitance and impedance of coplanar-strip waveguides on substrates with multiple dielectric layers," in Proceedings of the 2001 31st European Microwave Conference, EuMC 2001, gbr, September 2001.

[18] D. R. Smith, D. C. Vier, T. Koschny, and C. M. Soukoulis, "Electromagnetic parameter retrieval from inhomogeneous metamaterials," Physical Review E, vol. 71, Article ID 036617, pp. 142-154, 2005.

[19] X. Chen, T. M. Grzegorczyk, B.-I. Wu, J. Pacheco Jr., and J. A. Kong, "Robust method to retrieve the constitutive effective parameters of metamaterials," Physical Review E, vol. 70, Article ID 016608, 2004.

[20] D. R. Smith, W. J. Padilla, D. C. Vier, S. C. Nemat-Nasser, and S. Schultz, "Composite medium with simultaneously negative permeability and permittivity," Physical Review Letters, vol. 84, no. 18, pp. 4184-4187, 2000.

[21] W. J. Fu, Z. J. Qiu, Y. Y. Ying, M. Hua, Q. S. Bo, and X. Zhuo, “The design of left-handed metamaterials composed of magnetic resonators and electric resonators," Acta Physica Sinica, vol. 57, pp. 5015-5019, 2008.

[22] D. Ye, K. Chang, L. Ran, and H. Xin, "Microwave gain medium with negative refractive index," Nature Communications, vol. 5, p. $5841,2014$. 


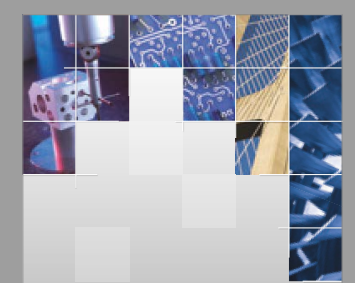

\section{Enfincering}
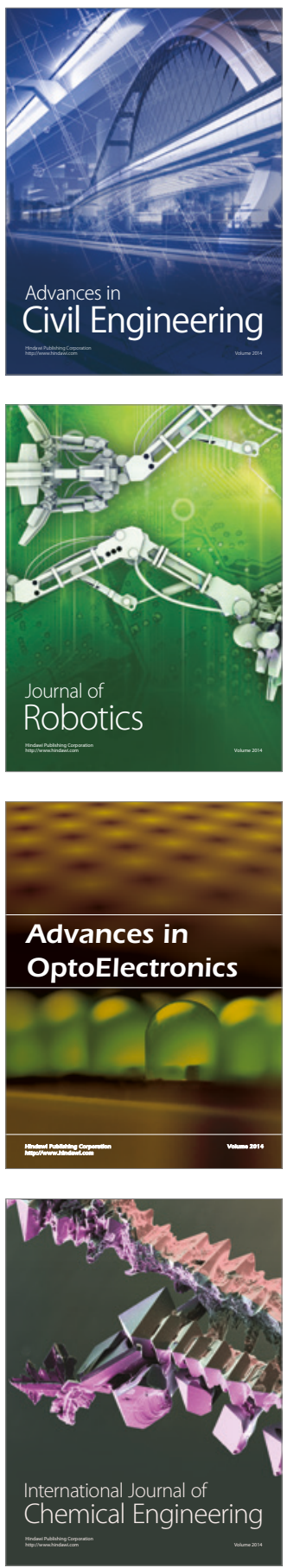

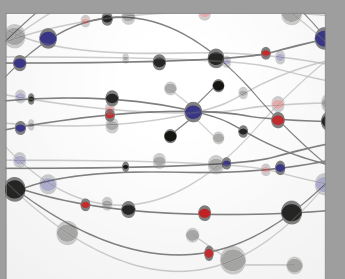

The Scientific World Journal

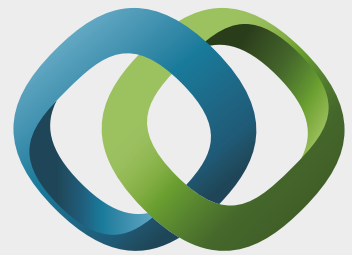

\section{Hindawi}

Submit your manuscripts at

https://www.hindawi.com
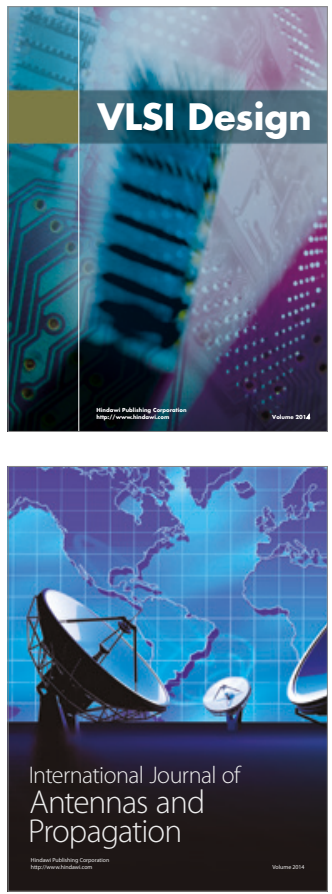

\section{Rotating}

Machinery
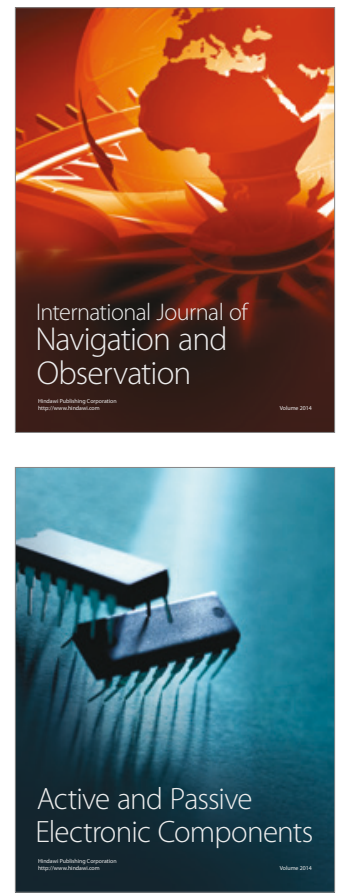
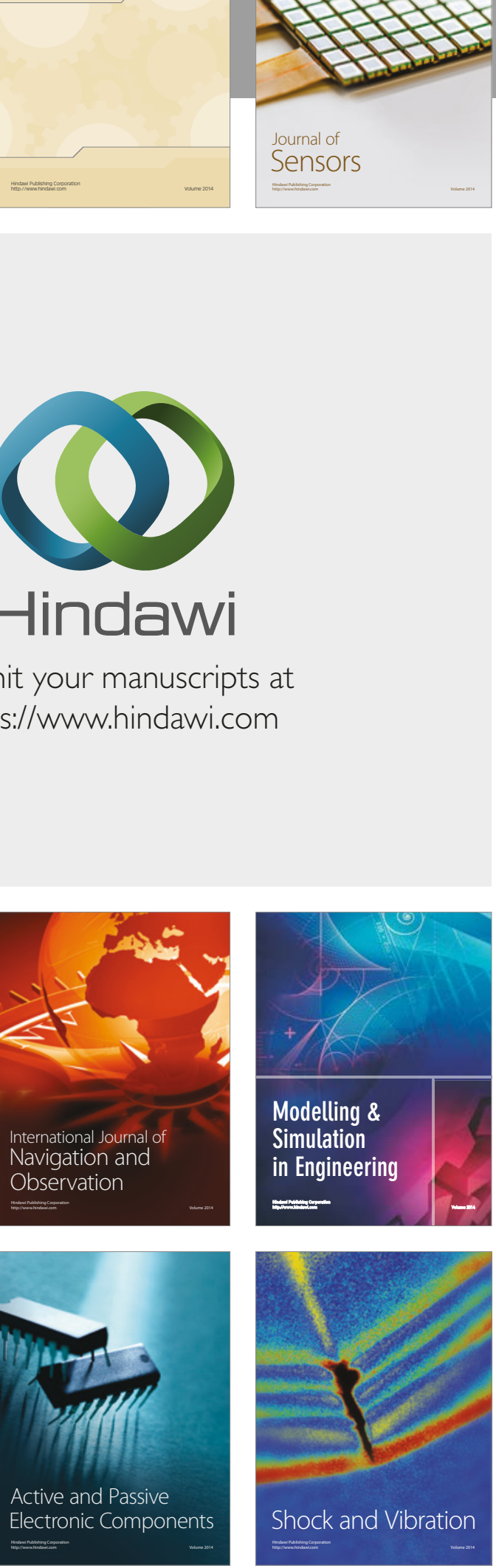
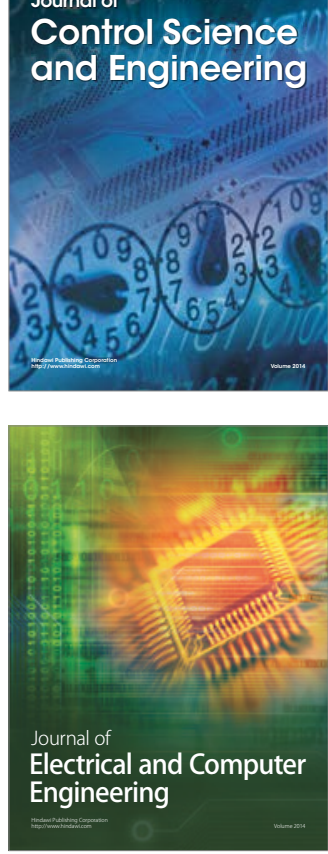

Distributed

Journal of

Control Science

and Engineering
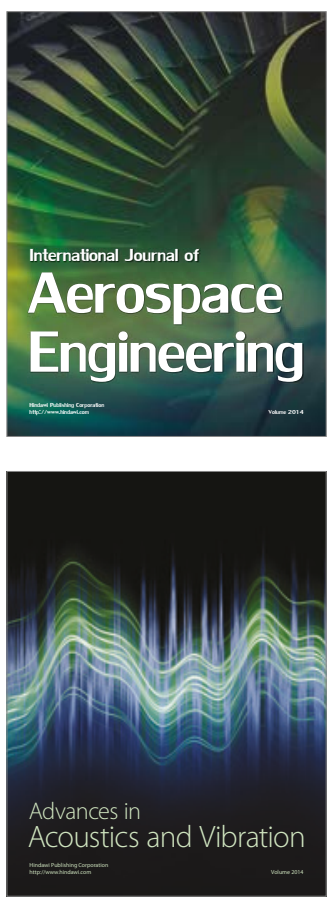

Sensor Networks 